\title{
Guía para referencia cardiológica de pacientes con síndrome inflamatorio multisistémico pediátrico y enfermedad por Kawasaki asociados al SARS-CoV-2
}

\section{Guide for cardiological referral for patients with paediatric multisystemic inflammatory syndrome and Kawasaki disease associated with SARS-CoV-2}

Humberto García-Aguilar*

Departamento de Cardiología Pediátrica, Centro Médico Nacional 20 de Noviembre, Instituto de Seguridad y Servicios Sociales de los Trabajadores del Estado, Ciudad de México, México

\begin{abstract}
Resumen
Presentamos una guía para la referencia al centro de atención especializada y el manejo inicial de pacientes pediátricos infectados por el coronavirus 2 del síndrome respiratorio agudo grave (SARS-CoV-2) con manifestaciones graves del síndrome multisistémico inflamatorio pediátrico o síntomas semejantes al síndrome de Kawasaki y que deben tener un abordaje multidisciplinario para garantizar un adecuado tratamiento y la mayor seguridad para el equipo de salud.
\end{abstract}

Palabras clave: Síndrome inflamatorio multisistémico pediátrico. Kawasaki. SARS-CoV-2.

\begin{abstract}
We present an institutional guide for a referral to the specialized care center and initial management of pediatric patients infected with severe acute respiratory syndrome coronavirus 2 (SARS-CoV-2) with severe manifestations of pediatric inflammatory multisystemic syndrome or symptoms similar to Kawasaki syndrome, and who must have a multidisciplinary approach to ensure adequate treatment and safety for the team of Health.
\end{abstract}

Key words: Pediatric multisystemic inflammatory syndrome. Kawasaki. SARS-CoV-2.

\section{Antecedentes}

La enfermedad por coronavirus 2019 (denominada COVID-19 por la Organización Mundial de la Salud [OMS] en enero de 2020) se debe a la infección por coronavirus 2 del síndrome respiratorio agudo grave (SARS-CoV-2) ${ }^{1}$. Este nuevo agente infeccioso para el ser humano tiene su origen aparente en la ciudad china de Wuhan, donde se detectó por primera vez en diciembre de 2019 en pacientes que presentaron disnea, tos, fiebre e infiltrados pulmonares. Fue hasta un mes después que se pudo identificar el virus responsable ${ }^{2}$.
Correspondencia:

*Humberto García-Aguilar

E-mail: humgaa@yahoo.com
Fecha de recepción: 03-06-2020

Fecha de aceptación: 20-08-2020

DOI: $10.24875 /$ ACM.200002581
Disponible en internet: 01-03-2021 Arch Cardiol Mex. 2021;91(Supl):40-46 www.archivoscardiologia.com 1405-9940 / @ 2020 Instituto Nacional de Cardiología Ignacio Chávez. Publicado por Permanyer. Este es un artículo open access bajo la licencia CC BY-NC-ND (http://creativecommons.org/licenses/by-nc-nd/4.0/). 


\section{COVID-19 y el paciente pediátrico}

Existen datos limitados que detallen los efectos de COVID-19 en la población pediátrica. Una revisión de 72,314 casos realizada por el centro chino para el control y prevención de enfermedades mostró que $<1 \%$ de los casos de COVID-19 se presentó en niños menores de 10 años $^{3}$. En otro estudio epidemiológico de 2,143 pacientes pediátricos evaluados por COVID-19 se confirmaron 731 casos mediante pruebas de reacción en cadena de la polimerasa con transcriptasa inversa (RT-PCR). Aunque se vieron afectados todos los grupos etarios pediátricos, la edad promedio fue de 7.0 años (rango: 1 día - 18 años). El 56\% de los pacientes pediátricos infectados eran de sexo masculino 4 .

Los niños diagnosticados con COVID-19 parecen tener una enfermedad menos grave en lo que hasta el momento se encuentra reportado ${ }^{5}$. Más del $90 \%$ de los casos hasta ahora descritos han sido de naturaleza leve o moderada. Sin embargo, los niños pequeños, especialmente los lactantes menores, parecen ser más susceptibles a las enfermedades graves por SARS-CoV-2 que los niños mayores ${ }^{6}$. En los últimos dos meses se han identificado un pequeño número de niños que desarrollan una respuesta inflamatoria multisistémica grave. Estos niños afectados gravemente llegaron a requerir cuidados intensivos pediátricos y la atención de equipos multidisciplinarios de especialistas. En una publicación de Bérgamo (Italia) ${ }^{7}$ se compararon retrospectivamente dos periodos de tiempo de casos pediátricos semejantes a la enfermedad de Kawasaki (Kawasaki-like), del 1 de enero de 2015 al 17 de febrero de 2020, y del 18 de febrero al 20 de abril de 2020. En este estudio se documentó un incremento de 30 veces el número de casos respecto al periodo anterior. La RT-PCR para SARS-CoV-2 fue positiva en dos de ellos, la inmunoglobulina G ( $\lg G)$ positiva en el $80 \%$, mientras que la lgM solo en el $30 \%$. El ecocardiograma fue anormal en el $60 \%$ de ellos y 2 pacientes tuvieron aneurismas coronarios mayores de 4 milímetros.

Este síndrome raro comparte características comunes con otras afecciones inflamatorias pediátricas que incluyen: enfermedad de Kawasaki, síndromes de shock tóxico por estafilococos y estreptococos, sepsis bacteriana y síndromes de activación de macrófagos. También puede presentarse con síntomas abdominales agudos y marcadores inflamatorios elevados ${ }^{8}$.

Considerando la gravedad de esta nueva entidad, es necesario el reconocimiento oportuno por parte de los médicos que atienden niños y la referencia temprana para atención especializada. Otro aspecto que resaltar es el exceso de información, las recomendaciones cambiantes y los nuevos conocimientos que se generan a diario, lo que hace muy difícil establecer protocolos de manejo claros y sobre todo seguros para los pacientes pediátricos afectados por el SARS-CoV-2.

\section{El Centro Médico Nacional 20 de Noviembre y la pandemia por COVID-19 en México}

A finales del mes de febrero de 2020, el Centro Médico Nacional 20 de Noviembre (CMN20N) se reconvirtió en Hospital COVID-19 por necesidad de atención especializada para este grupo de enfermos de nuestra institución, cuando la pandemia en nuestro país se encontraba por iniciar la fase II. La atención de enfermos infectados por SARS-CoV-2 desde la reconversión del CMN20N ha sido predominantemente para pacientes adultos, sin embargo, la presencia de un brote en pacientes pediátricos hospitalizados obligó a crear un protocolo específico para el tratamiento de los niños.

En el momento de la publicación en principios de junio de 2020 en México se reportaron 93,435 pacientes confirmados para COVID-19 y 10,167 muertes por esta causa. En datos oficiales del gobierno de México para mediados de mayo se reportaron los resultados del estudio de 6,942 enfermos menores de 18 años sospechosos; de esta cohorte 6,212 pacientes resultaron negativos, lo que indica un índice de positividad del 10.5\%, manteniendo la proporción de 1 de cada 10 casos sospechosos en niños con resultado positivo, como se ha publicado en otros trabajos ${ }^{9}$. En este mismo reporte, el 56\% (406) correspondió al sexo masculino. Respecto al tipo de atención que requirieron, el $80 \%$ (584) fueron pacientes ambulatorios. El $20 \%$ de los pacientes infectados (146) necesitaron hospitalización. El desarrollo de neumonía se registró en el $50 \%$ (73) de los niños hospitalizados; solo 12 niños fueron ingresados a una unidad de cuidados intensivos pediátricos, representando el $1.6 \%$ de los casos pediátricos totales de COVID-19. Del total de pacientes con prueba positiva en edad pediátrica, en el $52 \%$ (381) se identificó un contacto con prueba positiva para SARS-CoV-29.

Por otra parte, el inicio de reportes en otras ciudades del mundo de niños con datos clínicos de una enfermedad similar al síndrome de Kawasaki (SK) asociado a infección por SARS-CoV-2 nos alertó, al ser un centro de referencia de cardiología del Instituto de Seguridad y Servicios Sociales de los Trabajadores del Estado 
Tabla 1. Perfil clínico, imagen y de laboratorio

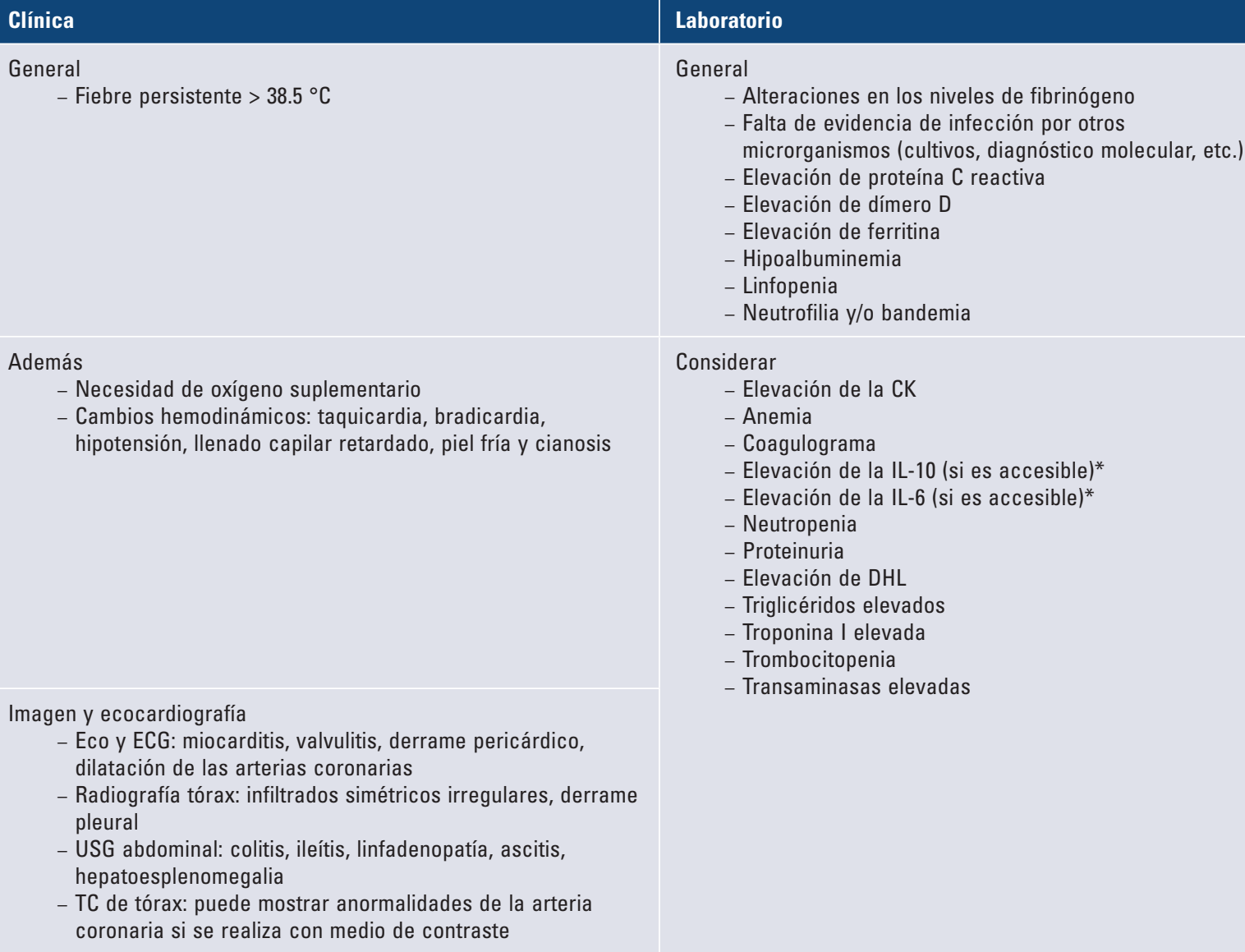

General

- Alteraciones en los niveles de fibrinógeno

- Falta de evidencia de infección por otros

microrganismos (cultivos, diagnóstico molecular, etc.)

- Elevación de proteína C reactiva

- Elevación de dímero D

- Elevación de ferritina

- Hipoalbuminemia

- Linfopenia

- Neutrofilia y/o bandemia

Considerar

- Elevación de la CK

- Anemia

- Coagulograma

- Elevación de la IL-10 (si es accesible)*

- Elevación de la IL-6 (si es accesible)*

- Neutropenia

- Proteinuria

- Elevación de DHL

- Triglicéridos elevados

- Troponina I elevada

- Trombocitopenia

- Transaminasas elevadas

*Estas pruebas no están ampliamente disponibles. La PCR se puede usar como un marcador sustituto de IL-6.

Eco: ecocardiograma; ECG: electrocardiograma; USG: ultrasonido; TC: tomografía computarizada; DHL: deshidrogenasa láctica; CK: creatinacinasa; IL: interleucina.

(ISSSTE), sobre cómo deberíamos abordar a estos niños para su diagnóstico, manejo y prevención de contagio a familiares y personal de salud ${ }^{10}$.

Este documento se desarrolló como una guía clínica, para brindar asesoría de gestión a los médicos que atienden niños en el ISSSTE y que necesitan derivar a este grupo de enfermos para evaluación por el servicio de cardiología del CMN20N.

\section{Principios generales sobre el tratamiento}

\section{Definición de caso}

Niños (0-18 años) que presentan fiebre persistente, inflamación sistémica documentada (neutrofilia, proteína $C$ reactiva elevada y linfopenia) y evidencia de disfunción de uno o varios órganos (choque cardiogénico, trastorno respiratorio, renal, gastrointestinal o neurológico) con características adicionales (Tabla 1).

\section{Kawasaki asociado a SARS-CoV-2}

Debe sospecharse SK asociado a SARS-CoV-2 en pacientes escolares y adolescentes (a diferencia del SK clásico, que se da en niños mas pequeños), que presentan fiebre prolongada (más de 5 días) con criterios similares al SK clásico completos o incompletos. El niño con sospecha de SK asociado a SARS-CoV-2 tiene con más frecuencia compromiso miocárdico (miocarditis o pericarditis) y no hay una respuesta adecuada a la primera dosis de inmunoglobulina intravenosa (IGIV), considerado como resistencia, por lo que requieren una segunda o tercera dosis de IGIV ${ }^{11}$.

Exclusión de cualquier otra etiología microbiológica, incluida la sepsis bacteriana, los síndromes de choque estafilocócicos o estreptocócicos, las infecciones asociadas con la miocarditis como el enterovirus (la espera de resultados de estudios de laboratorio no debe retrasar la búsqueda de asesoramiento experto) ${ }^{12}$. 
Las pruebas de PCR de SARS-CoV-2 pueden ser positivas o negativas ${ }^{13}$.

\section{Consideraciones especiales}

Todos los niños, sospechosos o confirmados, deben ser presentados lo antes posible para garantizar un tratamiento adecuado y seguro por un equipo multidisciplinario de salud.

Revisar la tabla 1 para obtener más características clínicas y de diagnóstico y para los estudios iniciales recomendados.

\section{Manejo médico temprano, protección personal y preservación de la vida.}

- Use del equipo de protección personal (EPP) apropiado y obligado. La gravedad de la enfermedad nunca sobrepasará la omisión de la protección del personal de salud, familiares y otros enfermos.

- Reanimación vital estándar y gestión para referencia a hospitalización o unidad de cuidados críticos de un centro de atención especializada.

- Tomar muestras para laboratorio según lo indicado en la tabla 2 y guardar suero para incluir estudios de investigación para confirmación de infección por SARS-CoV-2.

- El niño puede tener un deterioro rápido, por lo que el monitoreo cardiorrespiratorio no invasivo incluyendo saturaciones continuas, trazo electrocardiográfico (ECG) y toma de presión arterial, debe instalarse lo más temprano posible.

- Solicitar de forma inmediata ECG y ecocardiografía si es posible (el tiempo se determina por cuadro clínico).

- El diagnóstico ecocardiográfico de pacientes con sospecha de enfermedad de Kawasaki asociada a SARS-CoV-2 siempre deberá realizarse con EPP, si es posible con un equipo ecocardiográfico exclusivo para el área COVID-19, y/o tener siempre las precauciones de limpieza y desinfección apropiadas. En el caso de necesitar estudio ecocardiografía transesofágico, las recomendaciones deben extremarse. En este escenario es razonable partir de que el riesgo infeccioso es alto, siempre considerando que estos niños puedan ser positivos y que la intubación endotraqueal generará una gran carga de partículas virales en aerosol. En este contexto, el manejo de la vía aérea y la colocación de la sonda probablemente deberían proceder con las máximas precauciones de barrera. También es razonable que el equipo de la vía aérea (anestesiólogo o especialista en cuidados del enfermo en estado crítico) coloque y desplace la sonda transesofágica durante el estudio para minimizar los riesgos infecciosos de los operadores ${ }^{14}$.

- Si el paciente permanece en la unidad médica de primer contacto, los servicios locales deben brindarle apoyo continuo:

- Identificar compromiso multisistémica (hepático, renal, neurológico, hematológico, etc.).

- Tomar muestras para identificar infección por SARS-CoV-2 adicionales, incluidas muestras de sangre e hisopos previo al tratamiento con inmunoglobulina i.v.; si es urgente, iniciar antes de la referencia al centro de atención especializada.

\section{Consideraciones especiales}

Considerar IGIV y aspirina de forma temprana si cumple con los criterios para la enfermedad de Kawasaki y vigilar la probable resistencia y necesidad de una 2. ${ }^{\mathrm{a}}$ y $3 .^{\mathrm{a}}$ dosis $^{10-12}$. Considere IGIV si cumple con los criterios para el síndrome de shock tóxico ${ }^{10-12}$.

No utilice cloroquina/hidroxicloroquina de primera instancia ante los riesgos de arritmias fatales y de acuerdo con las nuevas recomendaciones internacionales ${ }^{15}$.

Todos los casos con sospecha de compromiso miocárdico (elevación de troponina I en suero y de péptido natriurético tipo $B$, así como cambios en el ECG y/o anormalidades del ecocardiograma) deben transferirse al centro de atención especializada en cardiología pediátrica.

\section{Seguimiento y monitoreo}

- Registro de signos vitales horarios y un conjunto completo de observaciones como gasto urinario, presión venosa central si es posible, uso de oximetría y capnografía no invasivas si estas son accesibles hasta considerar al paciente estable por más de 12 horas.

- Vigilar de cerca los signos de deterioro respiratorio, neurológico y cardiovascular.

- Monitoreo de signos clínicos de respuesta inflamatoria sistémica: empeoramiento de la fiebre, deterioro cardiorrespiratorio, empeoramiento de los síntomas gastrointestinales.

Otros datos de deterioro son: presencia de hepatoesplenomegalia, linfadenopatía, erupción generalizada y empeoramiento de los síntomas neurológicos.

Solicitar paraclínicos de apoyo en búsqueda de datos de inflamación progresiva mediante proteína $\mathrm{C}$ reactiva, 
Tabla 2. Estudios iniciales (la frecuencia de repetición depende de la necesidad clínica)

\begin{tabular}{l|l} 
Pruebas de laboratorio y microbiología. & Resultado comparado con valores para edad/sexo
\end{tabular}

Fecha/hora de la toma

Cultivos y/o pruebas moleculares

Serología viral

PCR para EBV, CMV, adenovirus, enterovirus

Glucosa

Gasometría arterial con lactato

Coagulación + fibrinógeno

Dímero D

DHL

Triglicéridos

Ferritina

Troponina

NT-Pro-BNP/BNP

CK

Vitamina D

Amilasa

Examen general de orina

Muestra de suero para PCR SARS-CoV-2 y

serología (idealmente antes de la IGIV)

Hemocultivo

Urocultivo

Cultivos respiratorios

Panel para SARS-CoV-2 PCR

PCR para neumococo, meningococo, estreptococo del grupo A,

Staphylococcus aureus, etc.

Otros

Serología SARS-CoV-2 si es accesible

$(\lg G, \lg M)$

Prueba de tuberculina o revisión de cicatriz de vacuna de BCG

PCR: reacción en cadena de la polimerasa; EBV: virus de Epstein-Barr; CMV: citomegalovirus; DHL: deshidrogenasa láctica; NT-ProBNP: porción terminal N del propéptido nutraceútico tipo B; BNP: péptido natriurético tipo B; CK: creatinacinasa; IGIV: inmunoglobulina intravenosa; BCG: vacuna del bacilo Calmette-Guérin; IgG: inmunoglobulina G; IgM: inmunoglobulina M.

procalcitonina, velocidad de sedimentación globular, disminución de los recuentos de células sanguíneas, ferritina en aumento, fibrinógeno alto o bajo, alteración de las pruebas funcionales hepáticas (aumento de alanina aminotransferasa, aspartato aminotransferasa o deshidrogenasa láctica), elevación de triglicéridos, incremento del dímero $\mathrm{D}$ y bajo contenido de sodio sérico acompañado de empeoramiento de la función renal.

\section{Busque el asesoramiento continuo del centro especializado y considere la transferencia si se produce un deterioro} (Fig. 1)

Principios generales sobre el tratamiento:

- Todos los niños deben ser tratados como sospechosos de COVID-19 


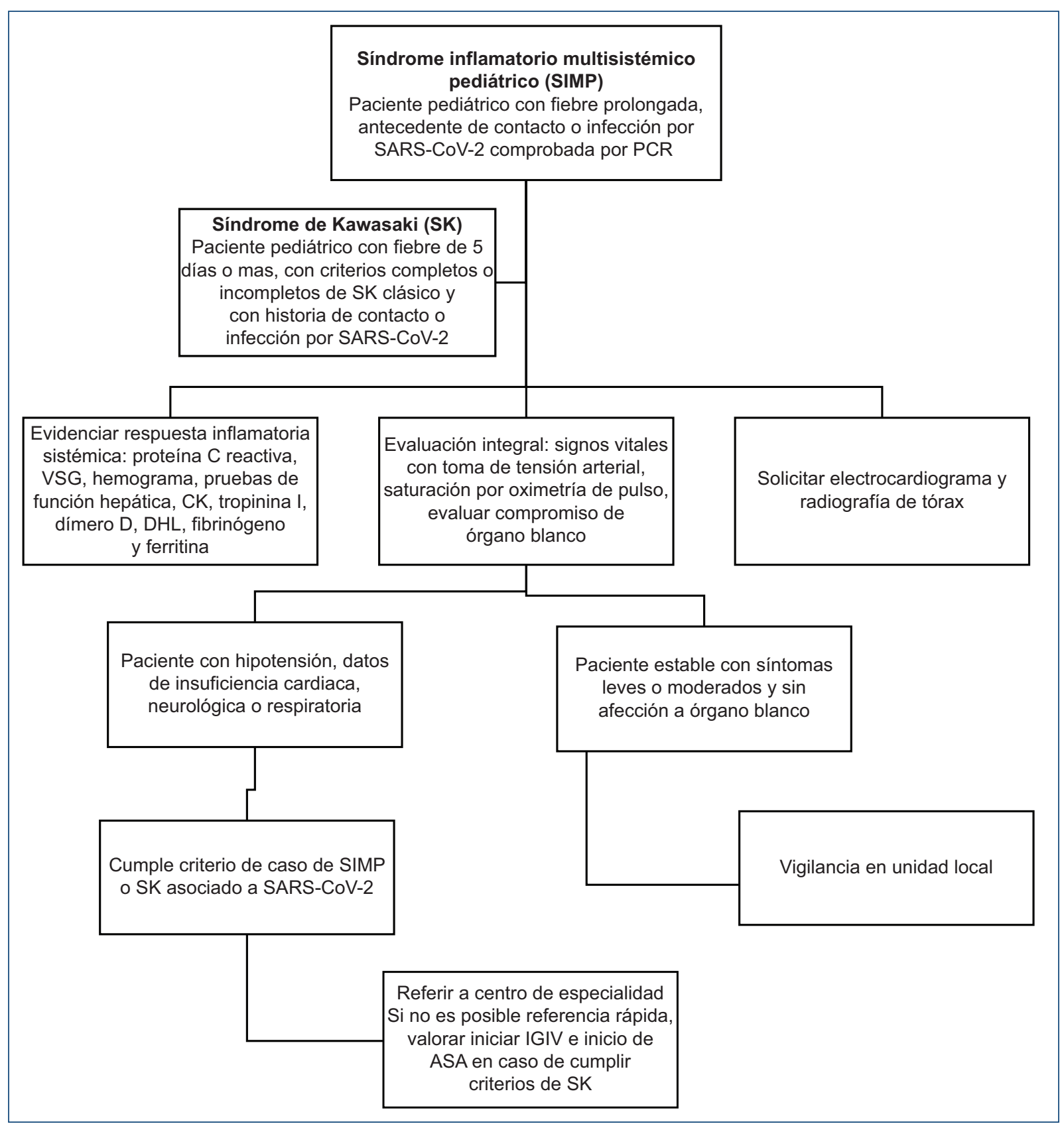

Figura 1. Algoritmo para evaluar al paciente sospechoso o confirmado de síndrome inflamatorio multisistémico 0 síndrome de Kawasaki (SK) asociado a SARS-CoV-2 y su referencia a hospital especializado.

SARS-CoV-2: coronavirus 2 del síndrome respiratorio agudo grave; PCR: reacción en cadena de la polimerasa; SVG; velocidad de sedimentación globular; DHL: deshidrogenasa láctica; CK: creatinacinasa; IGIV: inmunoglobulina intravenosa; ASA: ácido acetilsalicílico.

- Consulte las formas sobre la gestión de traslado de pacientes con COVID-19 o la sospecha de COVID-19 a la unidad especializada.

- Para enfermedades leves a moderadas, solo se recomienda la atención de apoyo.

- Si la enfermedad se presenta con deterioro rápido o es considerada clínicamente grave, discuta la transferencia a una unidad de cuidados intensivos con apoyo de Cardiología Pediátrica.

- Como esta es una condición de rápida evolución, todos los niños deben ser considerados para protocolos de tratamiento para COVID-19 locales. Sin embargo, se deben tomar en cuenta que este grupo de edad tiene condiciones diferentes, por lo que se 
deben ajustar estos protocolos con apoyo de médicos especialistas en pediatría.

- La terapia con inmunomoduladores (IGIV) debe discutirse con los médicos con experiencia adecuada en su uso ( $p$. ej., reumatología, inmunología, hematología) caso por caso y usarse en el contexto de un ensayo si es elegible y si está disponible.

\section{Consideraciones especiales}

El uso de corticosteroides, cloroquina, hidroxicloroquina, interferón, macrólidos, así como terapias antivirales, deben ser utilizadas en un contexto multidisciplinario de especialistas en inmunología, infectología y reumatología, ante la poca experiencia e información en la literatura sobre tratamiento en población pediátrica. Y siempre hay que considerar el riesgo-beneficio de la presentación grave de la enfermedad ${ }^{15}$.

\section{Conclusiones}

Esta guía se ha diseñado para la referencia de pacientes a un centro de atención cardiológica pediátrica del ISSSTE, con la intención de reducir los riesgos del personal de salud que atiende a estos enfermos, asegurar un diagnóstico y tratamiento óptimos a los futuros niños infectados con el SARSCoV-2 que pudieran presentar un síndrome inflamatorio multisistémico grave o un SK asociado a SARS-CoV-2 y coordinar un manejo multidisciplinario, ya que aún faltan muchas consideraciones fisiopatológicas por saber sobre este nuevo agente microbiológico y el impacto de la salud de los seres humanos ${ }^{16}$.

A la fecha existe mucha controversia sobre los tratamientos adecuados y las recomendaciones que en un principio son generales ante las nuevas evidencias en corto tiempo se contraindican, por lo que nuestra recomendación es ser cautelosos con el uso de fármacos y considerar un equipo multidisciplinario para estas decisiones que incluya a inmunólogos, reumatólogos, cardiólogos y especialistas en cuidados críticos $^{17-19}$.

\section{Financiamiento}

La presente investigación no ha recibido beca específica de agencias de los sectores público, comercial o sin ánimo de lucro.

\section{Conflicto de intereses}

Los autores declaran no tener conflicto de intereses.

\section{Responsabilidades éticas}

Protección de personas y animales. Los autores declaran que para esta investigación no se han realizado experimentos en seres humanos ni en animales.

Confidencialidad de los datos. Los autores declaran que en este artículo no aparecen datos de pacientes.

Derecho a la privacidad y consentimiento informado. Los autores declaran que en este artículo no aparecen datos de pacientes.

\section{Bibliografía}

1. Sohrabi C, Alsafi Z, O'Neill N, Khan M, Kerwan A, Al-Jabir A, et al. World Health Organization declares global emergency: A review of the 2019 novel coronavirus (COVID-19). Int J Surg. 2020;76:71-76.

2. Cucinotta D, Vanelli M. WHO Declares COVID-19 a Pandemic. Acta Biomed. 2020;91(1):157-60.

3. Lu H, Stratton CW, Tang YW. Outbreak of pneumonia of unknown etiology in Wuhan, China: The mystery and the miracle. J Med Virol. 2020;92:401-2.

4. Wu Z, McGoogan JM. Characteristics of and important lessons from the coronavirus disease 2019 (COVID-19) outbreak in China: summary of a report of 72314 cases from the Chinese Center for Disease Control and Prevention. JAMA. 2020;323(13):1239-42.

5. Lu X, Zhang L, Du H, Zhang J, Li YY, Qu J, et al.; Chinese Pediatric Novel Coronavirus Study Team. SARS-CoV-2 infection in children. N Engl J Med. 2020;382(17):1663-5.

6. Zimmermann $\mathrm{P}$, Curtis $\mathrm{N}$. Coronavirus infections in children including COVID-19: An overview of the epidemiology, clinical features, diagnosis, treatment and prevention options in children. Pediatr Infect Dis J. 2020;39(5):355-68.

7. Shekerdemian LS, Mahmood NR, Wolfe KK, et al. Characteristics and outcomes of children with coronavirus disease 2019 (COVID-19) infection admitted to US and Canadian Pediatric Intensive Care Units. JAMA Pediatr. 2020;174(9):868-73.

8. CDC COVID-19 Response Team. Coronavirus Disease 2019 in Children - United States, February 12-April 2, 2020. MMWR Morb Mortal Wkly Rep. 2020;69:422-6.

9. https://datos.gob.mx/busca/dataset/informacion-referente-a-casos-covid-19-en-mexico

10. Shulman ST. Pediatric COVID-associated Multi-system Inflammatory Syndrome (PMIS). J Pediatric Infect Dis Soc. 2020;9(3):285-6

11. Deza Leon MP, Redzepi A, McGrath E, Abdel-Haq N Shawaqfeh A Sethuraman U, et al. COVID-19 associated pediatric multi-system inflammatory syndrome. J Pediatric Infect Dis Soc. 2020;9(3):407-8.

12. Rauf A, Vijayan A, John ST, Krishnan R, Latheef A. Multisystem inflammatory syndrome with features of atypical Kawasaki disease during COVID-19 pandemic. Indian J Pediatr. 2020;87(9):745-7.

13. Harwood R, Sinha I. Diagnosis of COVID-19 in children: the story evolves. BMC Med. 2020;18(1):158.

14. Augoustides JG. Perioperative echocardiography during the coronavirus crisis: Considerations in pediatrics and congenital heart disease. J Cardiothorac Vasc Anesth. 2020;34(9):2303-6.

15. La FDA advierte en contra del uso de la hidroxicloroquina o la cloroquina para el tratamiento del COVID-19 fuera del entorno hospitalario o de un ensayo clínico debido al riesgo de problemas con el ritmo cardíaco [Internet]. EE.UU: Food and Drug Administration, Aviso de seguridad; 04-24-2020. Disponible en: https://www.fda.gov/drugs/drug-safety-and-availabilityla-fda-advierte-en-contra-del-uso-de-la-hidroxicloroquina-o-la-cloroquina-para-el-tratamiento-del

16. Yuki K, Fujiogi M, Koutsogiannaki S. COVID-19 pathophysiology: A review. Clin Immunol. 2020;215:108427.

17. Nguyen AA, Habiballah SB, Platt CD, Geha RS, Chou JS, McDonald DR Immunoglobulins in the treatment of COVID-19 infection: Proceed with caution! Clin Immunol. 2020;216:108459.

18. Jean SS, Lee PI, Hsueh PR. Treatment options for COVID19: The reality and challenges. J Microbiol Immunol Infect. 2020;53(3):436-43. 